\title{
A validated porcine corneal organ culture model to study the limbal response to corneal epithelial injury
}

\author{
Ashkon G. Seyed-Safi ${ }^{1,2,3}$, Julie T. Daniels ${ }^{1}$. \\ ${ }^{1}$ UCL Institute of Ophthalmology, ${ }^{2}$ UCL Medical School. \\ ${ }^{3}$ Corresponding author: ashkon.seyed-safi.16@ucl.ac.uk \\ Address: UCL Institute of Ophthalmology, 11-43 Bath street, EC1V 9EL, London, UK
}

Declaration of interests: none.

\begin{abstract}
Limbal epithelial stem cells are required for the maintenance and repair of the cornea epithelial surface. The difficulty in obtaining human corneal tissue for research purposes means that animal models for studying the corneal and limbal epithelium are extremely useful. In particular, in organ culture systems, animal models can enable the study of limbal epithelial stem cells in the context of their native niche. The similarities of the porcine limbal microanatomy and stem cell marker expression make it an attractive model, however, functional analysis of the limbal epithelial cell population is needed to validate the use of the tissue. Single cell clonal analysis revealed that holoclone-generating cells were enriched in the limbus as compared with the central cornea $(38.3 \%$ vs $8.3 \%)$ and that label-retaining cells were also enriched in the limbus and compared with the central cornea (44.7 \pm 6.4 vs $4.7 \pm 1.5)$. Furthermore, it was demonstrated that in a 3D-printed organ culture system, porcine tissue was capable of maintaining and healing the corneal epithelium. Ki67 staining of corneal sections revealed a proliferative response in the limbal basal epithelium that subsequently resolved, in response to central epithelial wounding. Therefore, the authors present a comprehensively validated model system which will be a powerful tool in studying the interactions between limbal niche factors and limbal epithelial stem cell fate.
\end{abstract}




\section{INTRODUCTION}

1.1 The epithelium is the outermost layer of the cornea and its integrity is a key determinant of corneal function. In humans it is widely accepted that corneal epithelium is maintained by a population of unipotent epithelial stem cells (Limbal epithelial stem cells, LESCs) concentrated in the limbal stem cell niche, located at the corneoscleral junction (Dziasko et al., 2014; Dziasko and Daniels, 2016; Pellegrini et al., 1999; Shortt et al., 2007; Thoft and Friend, 1983). A greater understanding of the factors involved in regulating stem cell fate is necessary for further development of treatments within this field; both to enhance the efficacy of ex vivo LESC expansion, and in revealing new therapeutic strategies based around reconstructing the niche (Yazdanpanah et al., 2019).

To further understand the role of the limbal niche microenvironment and the contribution of individual niche factors to the regulation of LESC fate, it is important to progress from $2 \mathrm{D}$ in vitro studies, to being able to study these cells in a 3D tissue context. One challenge is the difficulty in obtaining suitable numbers of whole human corneas of sufficient quality, in which the relationship between these factors and LESC function can be studied. Therefore, animal models of the human cornea and limbus are invaluable in providing insights into LESC biology.

Seminal lineage tracing studies in vivo in mice have been important in furthering our understanding of LESC behaviour in homeostasis and under wounding conditions (Amitai-Lange et al., 2015; Di Girolamo et al., 2015; Dorà et al., 2015). However, the ethical and practical considerations that come with using live animals mean that there is increasing interest in developing ex vivo models. With these models, cells can be studied in their whole-tissue context, whilst sidestepping the many technical challenges of in vivo studies (Stepp et al., 2014). This approach also incorporates the principles of Replacement, Reduction, and Refinement in animal research, first outlined by Russell and Burch in 1959 (Tannenbaum and Bennett, 2015).

Accordingly, while organ culture systems have been used to study the behaviour of the corneal epithelium in research-donated human eyes (Chang et al., 2008; Saghizadeh et al., 2010), there are also many examples of corneal tissue from animals being used instead, including from dogs (Proietto et al., 2017), cows (Foreman et al., 1996; Zhao et al., 2006) and pigs (Barrera et al., 2018; Notara et al., 2011). An important consideration in such investigations is whether the corneal epithelial cell biology of other species is suitably similar to that of humans.

While the mouse is well-suited for in vivo studies, there are significant differences between the human and murine limbus (Li et al., 2017). One in particular is the lack of limbal crypt structures that are believed to represent a key aspect of the human limbal niche (Dziasko et al., 2014). In contrast, the porcine ocular surface has been shown to have a great deal of similarity with the human ocular surface (Crespo-Moral et al., 2020) and indeed limbal interpalisade crypt structures have been demonstrated in porcine tissue (Notara et al., 2011; Grieve et al., 2015; Patruno et al., 2017). Additionally, studies have demonstrated a functional compatibility between human and porcine epithelial tissue (Notara et al., 2011). However, the authors believe that further investigations, including single cell clonal analysis and label-retention studies, are important to further validate the suitability of this tissue as a model for studying LESC behaviour.

This article presents work that has been carried out to further validate the porcine cornea as a suitable model for studying limbal stem cell behaviour, and the subsequent development of an ex vivo organ culture model in which the behaviour of the limbal epithelium can be studied in response to central corneal epithelial damage. In assessing the suitability of this porcine model, the key hypothesis was that as in human tissue, cells with a stem cell phenotype are enriched within the limbus. This was investigated with respect to the microanatomy of the porcine limbus as well as its staining profile of key LESC markers. Furthermore, reflecting many of the early studies in human tissue, clonal analysis and label-retention studies of the corneal and limbal epithelium were also performed. The results confirm the similarities in the limbal epithelial phenotype between humans and pigs.

Building on many of the organ culture models that already exist, 3D printed scaffolds were used to refine a practical and easy-to-maintain system. Key objectives were preservation of the multi-layered epithelium and limbal microanatomy; a consistent and conserved regenerative response to epithelial damage; evidence of the 
requirement for an intact limbal niche for epithelial function; and evidence of a limbal proliferative response to central corneal epithelial debridement. 


\section{MATERIALS AND METHODS}

\subsection{Corneal tissue}

Human limbal tissue was obtained from three donors and used for sectioning. All experiments were approved through the Moorfields Biobank Internal Ethics Committee (10/H0106/57-2011ETR10) and carried out according to the tenets of the Declaration of Helsinki.

Eyes from 6-8-month-old pigs were purchased as redundant tissue from Humphreys \& Sons (Blixes farm, Chelmsford, United Kingdom). Eyes were collected within 1 hour of extraction and transported to the lab on ice and processed immediately. Excess muscle, connective tissue, and conjunctiva were removed before the cornea was separated from the globe, with a small rim of scleral tissue remaining. Anterior chamber structures were separated from the corneoscleral rim (CSR), which was then washed: two minutes in 2\% (v/v) Povidone-Iodine solution, one minute in 1\% (v/v) Antibiotic-Antimycotic solution (ABAM; Sigma-Aldrich, Dorset, UK), and one minute in phosphate-buffered-saline (PBS).

\subsection{Visualising corneal sections}

Corneas were bisected along the horizontal meridian and submerged in OCT (optimal cutting temperature) compound in cryomolds before being frozen in liquid nitrogen. Cryosections, of 8-10 $\mu \mathrm{m}$ thickness, were cut from the resulting blocks using a Leica CM1850 cryostat (Leica microsystems, Milton Keynes, UK) and transferred onto superfrost plus microslides (Fisher Scientific, Loughborough, UK). Slides were left to dry before being stored at $-20^{\circ} \mathrm{C}$.

For visualising the anatomy, sections were fixed in $4 \%(\mathrm{v} / \mathrm{v})$ paraformaldehyde (PFA) before being stained with haematoxylin and eosin using an automated staining machine (Leica ST5010 Autostainer XL, Wetzlar, Germany). Sections were imaged using a Nikon Eclipse TS100 inverted microscope (Nikon Instruments Europe B.V., Surrey, UK).

For immunofluorescence, frozen sections were fixed with 4\% PFA for 10 minutes. Slides were washed three times with PBS. If the epitope was located intracellularly, cells were permeabilised with $0.5 \%(\mathrm{v} / \mathrm{v})$ Triton X100 in PBS for 7 minutes at room temperature. Slides were then washed three times with PBS once more. Tissue was blocked for 75 minutes in PBS supplemented with 5\% (v/v) goat serum. Sections were then incubated with the primary antibody in PBS with $5 \%$ goat serum, in a moistened chamber, overnight at $4{ }^{\circ} \mathrm{C}$. Antibodies were used against p63 (1:100, orb11214, Biorbyt, Cambridge, UK), cytokeratin 3 (1:200, CBL218, Merck Millipore UK, Watford, UK), and cytokeratin 15 (1:100, sc-47697, Santacruz, Texas, US).

For negative controls, IgG of the appropriate species, isotype, and concentration were added instead of the primary antibody for negative controls. Sections were then washed three time with PBS and incubated with the appropriate secondary antibody or counterstained with FITC conjugated phalloidin (1:500) in PBS with 5\% goat serum for one hour at room temperature. Slides were again washed three times with PBS and mounted using Vectashield medium with DAPI (Vector laboratories Ltd. Peterborough, UK) and coverslipped. Confocal images were captured using a Zeiss 710 confocal microscope (Carl Zeiss, Hertfordshire, UK). Images were captured at either 10X or 40X magnification with water immersion lenses, with an averaging of 16 scans. In order to balance the colours, generate overlays, and incorporate scale bars, images were processed using Image $\mathrm{J}$.

\subsection{Cell culture}

The corneoscleral rim was dissected to generate four arrow shaped segments, each including central and peripheral cornea, limbus and scleral tissue. Limbal and central corneal tissue was transferred to a solution containing 1.2 U/mL dispase II (Roche Diagnostics GmbH, Mannheim, Germany) in corneal epithelial culture medium (CECM) overnight at $4^{\circ} \mathrm{C}$. Following digestion, epithelial sheets were separated from the pieces of tissue and were treated with $0.25 \%(\mathrm{v} / \mathrm{v})$ TE-EDTA (Life Technologies, Paisley, UK) in PBS at $37^{\circ} \mathrm{C}$ for $25-30$ minutes, with regular agitation of the mixture to generate a single cell suspension, a result which was confirmed on microscopic examination.

Epithelial cells were seeded on growth arrested 3T3s (3T3s; a gift from Prof. Fiona Watt, Cancer Research, UK) and maintained in corneal epithelial cell culture medium (CECM) containing a 1:1 ratio of DMEM high glucose: F12, 10\% (v/v) fetal bovine serum, epidermal growth factor (EGF) $(10 \mathrm{ng} / \mathrm{mL})($ Life Technologies, 
Paisley, UK), adenine $(0.18 \mathrm{mM})$, transferrin $(5 \mu \mathrm{g} / \mathrm{mL}), \mathrm{T} 3(2 \mathrm{nM})$, cholera toxin $(0.1 \mathrm{nM})$, hydrocortisone $(0.4 \mu \mathrm{g} / \mathrm{mL})$, insulin $(5 \mu \mathrm{g} / \mathrm{mL}), 1 \%$ Antibiotic-Antimycotic (v/v) (Sigma-Aldrich, Dorset, UK). CECM media was changed three times a week, and co-cultures were maintained at $37^{\circ} \mathrm{C}$ in an incubator with $5 \% \mathrm{CO}_{2}$ in air. Cells were counted, 3T3 confluency assessed, and epithelial cell morphology was monitored using an inverted phase contrast microscope (Nikon Eclipse TS100 inverted phase contrast microscope, Nikon Instruments Europe B.V., Surrey, UK)

\subsection{Clonal analysis}

1500 epithelial cells derived from limbal or central corneal epithelial sheets were seeded on a layer of growth arrested $3 \mathrm{~T} 3 \mathrm{~s}$ in $55 \mathrm{~cm}^{2}$ culture plates and cultured for up to seven days. Once microscopic colonies could be identified five colonies were randomly and independently selected for clonal transfer. Culture media was aspirated, and each plate was washed with PBS. Polystyrene cloning cylinders (8mm) (Sigma-Aldrich, Dorset UK) were dipped in sterile vacuum grease (Sigma-Aldrich, Dorset UK) and attached to the plate surface surrounding each of the randomly selected colonies. Subsequently, 0.5\% TE-EDTA (Life Technologies, Paisley, UK) was added to the cloning cylinder for two minutes at $37^{\circ} \mathrm{C}$ to detach the epithelial cells which were then transferred to another $55 \mathrm{~cm}^{2}$ culture plate with a layer of growth arrested $3 \mathrm{~T} 3 \mathrm{~s}$; these were referred to as "indicator plates". 5 primary cultures were taken from each of the central and limbal epithelium of each eye. 4 Clones isolated at random were then transferred to indicator plates to be cultured for 12 days on 3T3s. Cells were cultured on indicator plates for 12 days. After 12 days, plates were fixed with 4\% PFA, before being stained with $1 \%$ Rhodamine B. Plates were then rinsed with $\mathrm{dH}_{2} \mathrm{O}$ (deionised water). In accordance with the methods outlined by Barrandon and Green (Barrandon and Green, 1987), plates were then classified as holoclones, meroclones, or paraclones, depending on the proportion of colonies on the plate that were aborted (terminally differentiated). For assessment, colonies on plates were examined microscopically using an inverted phase contrast microscope (Nikon Eclipse TS100 inverted phase contrast microscope, Nikon Instruments Europe B.V., Surrey, UK).

The results of clonal analysis of cells from the central corneal and limbal epithelium were expressed in a $3 \times 2$ contingency table and statistical significance was assessed using the Freeman-Halton extension of Fisher's exact probability test.

\subsection{Organ culture of corneas}

A scaffold for the corneoscleral rims (CSRs) was developed by modifying a standard 6-well tissue culture plate. A platform was designed using the free online tool, Tinkercad (www.tinkercad.com), and 3D-printed in autoclavable Nylon SLS (Selective laser sintering) (Digits2Widgets, London, UK). The platform consisted of a flat circular base, with four protruding pegs. Once autoclaved, platforms were attached to the plates using a cyanoacrylate glue, and the overall scaffold was left under UV light for one hour before use.

Once washed, the CSRs were inverted in a corneal viewing chamber (Bausch \& Lomb UK, Surrey, UK). An agar mixture, containing $1.5 \%(\mathrm{w} / \mathrm{v})$ agar in DMEM with $1 \%$ ABAM, was prepared and sterilised before being warmed to liquid state and added to fill the CSR concavity and left to set, in order to maintain the anterior chamber shape. Once set, the CSR filled with agar was attached to the scaffold and tethered by the pegs of the platform (figure 2.1).

For the first two days, CSRs were fed with an antibiotic fortified culture medium containing a 1:1 ratio of DMEM high glucose: F12, $10 \%(\mathrm{v} / \mathrm{v})$ fetal bovine serum, adenine $(0.18 \mathrm{mM})$, transferrin $(5 \mu \mathrm{g} / \mathrm{mL}), \mathrm{T} 3(2 \mathrm{nM})$, cholera toxin $(0.1 \mathrm{nM})$, hydrocortisone $(0.4 \mu \mathrm{g} / \mathrm{mL})$, insulin $(5 \mu \mathrm{g} / \mathrm{mL}), \mathbf{1 0} \%$ Antibiotic-Antimycotic $(\mathrm{v} / \mathrm{v})$ (Sigma-Aldrich, Dorset, UK).

Subsequently, corneas were fed with an sustained cultured medium containing a 1:1 ratio of DMEM high glucose: $\mathrm{F} 12,10 \%(\mathrm{v} / \mathrm{v})$ fetal bovine serum, adenine $(0.18 \mathrm{mM})$, transferrin $(5 \mu \mathrm{g} / \mathrm{mL})$, T3 $(2 \mathrm{nM})$, cholera toxin $(0.1 \mathrm{nM})$, hydrocortisone $(0.4 \mu \mathrm{g} / \mathrm{mL})$, insulin $(5 \mu \mathrm{g} / \mathrm{mL}), \mathbf{1 \%}$ Antibiotic-Antimycotic (v/v) (Sigma-Aldrich, Dorset, UK). The use of DMEM in the organ culture of corneal epithelium was in accordance with its use in previously published organ culture systems (Proietto et al., 2017). Medium was added dropwise daily to each well up to the level of the limbus. Organ cultured corneas were incubated at $37^{\circ} \mathrm{C}$ and $5 \% \mathrm{CO}_{2}$ inside an incubator on a 3D orbital rotating shaker (Grant Instruments, Cambridge, United Kingdom) at 24 cycles $/ \mathrm{min}$. 
In the instance of suspected infection, the affected cornea was disposed of along with any other tissue in the same 6-well plate scaffold.

\subsection{Wounding of organ culture corneas}

Excess moisture was removed from the surface of the corneas using absorbent spears, or towels. A blunted 8mm trephine was gently applied to the central surface of the cornea to demarcate a wound area and under the microscope a blunted crescent blade (REF) or hockey stick tool was used to debride the epithelium within the marked area (figure 3.3C).

Epithelial wound size was monitored by staining corneas with $2 \%$ 'Minims' fluorescein dye (Bausch \& Lomb UK, Surrey, UK). Media was aspirated from the wells, and the corneal surface was moistened with PBS, before a drop of fluorescein was added to the surface. 2-300mL of PBS was then added to the surface to disperse the dye. Corneas were illuminated using a Fiber-Lite MI-150 illuminator (Dolan-Jenner Industries, Boxborough MA, USA), and imaged with a camera phone, with time stamps on the photos to help track the rates of healing. Following this, the CSR was washed with media, and fresh media was once again added up to the level of the limbus.

Due to heterogeneity in epithelial integrity of eyes following transport and processing, a validation step was included before wounding studies. A central epithelial wound was created in the tissues at the time of being put in organ culture. Corneas were followed until the epithelial surface had healed, as indicated by the lack of surface fluorescein staining. Any corneas where the surface failed to heal was discarded from future wounding studies. Once healed, all corneas were left to stabilise for two days to achieve a consistnet baseline.

Subsequently, another $8 \mathrm{~mm}$ epithelial wound was created and wounded corneas were frozen for sectioning at 3 , $6,12,18,24,36,48,72$, and 168-hour time points.

\subsection{Label retention studies}

Organ cultured corneas were incubated for 72 hours with added BrdU at a concentration of $10 \mu \mathrm{M}$. The media was changed daily and at the end of this period the corneas were washed three times in PBS. The corneas were subsequently maintained in normal culture medium and cultured for either 24 hours, or 4 weeks before being frozen for sectioning. Sections were stained using a rat monoclonal antibody against $\operatorname{BrdU}(1: 100$, ab6326, Abcam, Cambridge, UK). In addition to the staining protocol described above, following the permeabilization step, a DNA denaturing step was included wherein slides were incubated with $2 \mathrm{~N} \mathrm{HCl}$ for 60 minutes at $37^{\circ} \mathrm{C}$, before being washed three times with PBS. Sections were visualised as described above.

\subsection{Scanning electron microscopy}

Following either debridement of the limbal and corneal epithelium, or debridement as well as treatment with $1 \mathrm{M}$ $\mathrm{N}$-heptanol for $120 \mathrm{~s}$, corneas were fixed overnight at $4^{\circ} \mathrm{C}$ in Karnovsky's medium, containing $2.5 \%$ gluteraldehyde, $1 \%$ paraformaldehyde, $80 \mathrm{mM}$ cacodylate buffer, and $20 \mathrm{mM} \mathrm{NaOH}$. Samples were washed three times for 5 minutes each with a wash buffer containing $1 \mathrm{M}$ sodium cacodylate. A $2 \%$ aqueous osmium tetroxide treatment was performed for 2 hours, followed by five 5 -minute washes with $\mathrm{ddH}_{2} 0$. Samples were then dried serially at room temperature with $50 \%, 70 \%, 90 \%$ ethanol for 15 minutes each, and then $100 \%$ ethanol and $100 \%$ methanol twice each for 30 minutes, and finally 100\% Hexamethyldisilazane once for 30 minutes, and once more overnight. At room temperature, $100 \%$ conductive silver paint was applied and left to dry overnight. Finally, gold/palladium sputter coating was applied to 1.5-1.7nM (Cressington 108 auto sputter coater, Watford, UK). Samples were imaged using a Zeiss Sigma VP field emission scanning electron microscope (Carl Zeiss, Hertfordshire, UK). 


\section{RESULTS}

\subsection{Porcine limbal epithelial phenotype}

Fresh porcine corneas $(n=3)$ were frozen and sectioned to examine the ultrastructure of the epithelium. Haematoxylin and Eosin staining of sections from the superior, nasal, inferior, and temporal regions of the limbus revealed that deep invaginations of the limbal epithelium, analogous to the limbal interpalisade crypts found in human limbal tissue, were present in sections from all regions (figure 3.1A)

Sections were also stained to establish the distribution of cells expressing markers associated with an LESC phenotype. Cells in the basal layer of the limbus, exclusively, were positive for expression of p63 and cytokeratin 15 (CK15). These cells were also negative for the expression of a marker of epithelial differentiation, cytokeratin 3 (CK3). This pattern was compared with that observed in human tissue and was found to be conserved (figure $3.1 \mathrm{~B} / \mathrm{C}$ ).

To identify slow-cycling cells within the tissue, porcine corneas $(n=3)$ were organ-cultured for 4 weeks following $72 \mathrm{~h}$ of culture in media containing $10 \mu \mathrm{M} \mathrm{BrdU}$. At the end of the chase period, corneas were frozen and sectioned, and stained with anti-BrdU antibody. The frequency of label-retaining cells was statistically significantly higher in the limbus as compared with the central cornea $(44.7 \pm 6.4$ in the limbal sections vs $4.7 \pm 1.5$ in the central cornea $(\mathrm{n}=3, P<0.05)$ ) (figure 3.1D).

The clonal capacity of epithelial cells from the limbus and central cornea was compared through single cell clonal analysis. The colonies on each indicator plate represents the progeny of a single clone that has been serially passaged. Each plate was classified as a holoclone, meroclone, or paraclone, according to the proportion of terminal colonies that were identified (Barrandon and Green, 1987).

Paired limbal and central samples were compared from three different eyes, with 20 indicator plates being generated from the limbal and central epithelial cells of each eye (figure 3.2). Of the 60 indicator plates derived from central epithelial clones, $5(8.3 \%)$ were classified as holoclones, $53(88.3 \%)$ as meroclones, and $2(3.3 \%)$ as paraclones. Of the 60 indicator plates derived from limbal epithelial clones, $23(38.3 \%)$ were classified as holoclones, $33(55 \%)$ as meroclones, and $4(6.7 \%)$ as paraclones. The association between the corneal and limbal results was assessed using the Freeman-Halton extension of Fisher's exact probability test (Lydersen et al., 2007). The two outcomes were found to be statistically significantly different at the $0.001 \%$ level $(P<0.0001)$

\subsection{Limbal epithelial function and phenotype under organ culture conditions}

Eyes were collected and processed within hours of enucleation and an $8 \mathrm{~mm}$ epithelial wound was created on the surface of the cornea. Following dissection of extra-corneal tissues, and washing of the corneoscleral rims, the tissues were kept in culture for 7-12 days (pre-treatment phase), during which time they regenerated a multilayered corneal epithelium. Corneas that did not demonstrate regeneration of the epithelial surface were excluded from subsequent experiments.

Under these conditions the crypt-like structures in the limbal epithelium were preserved, as were the limbal expression patterns of $\mathrm{p} 63$, CK15, and CK3 in the limbus. (figure 3.3B). The only difference observed was that, unlike in non-cultured tissue, p63-positive cells were also present in the basal layer of the corneal epithelium. Following the pre-treatment phase, an $8 \mathrm{~mm}$ in diameter region of the central corneal epithelium was again debrided. The wound area was confirmed with fluorescein staining, and closure of the wound was monitored regularly every $24 \mathrm{~h}$. Sections of healing corneas revealed that following wounding, an initial monolayer of epithelium was formed, followed later by stratification (figure 3.3D). In a series of 6 wounded corneas, the wound area detectable by fluorescein staining had healed completely by the $72 \mathrm{~h}$ timepoint. In addition, it was observed that the corneas were capable of healing multiple sequential wounds at a consistent rate (figure 3.4). The role of an intact limbal niche in this model was also investigated. Epithelium was completely debrided from the entire cornea and limbus of two corneas, and one of them was subsequently subjected to N-heptanol treatment. SEM images of each condition were generated, revealing that following debridement of the limbus the interpalisade crypt structures were preserved whereas in corneas treated with $\mathrm{N}$-heptanol this microanatomy was lost (figure 3.5A). The same conditions (debridement vs debridement and $\mathrm{N}$-heptanol) were applied to 8 corneas, and 4 of each condition were cultured for 5 days. Epithelium grew towards the centre from the limbus 
in the debridement-only corneas, but in the debridement-with-N-heptanol corneas, there was no detectable reepithelialisation (figure 3.5B)

\subsection{Limbal epithelial response to central corneal epithelial wounding}

In order to assess whether a limbal proliferative response to wounding was present in this model, corneas were frozen and sectioned at a series of time points after wounding (3h,12h, 24h, 48h, 72h, and 1 week after wounding). Sections were stained for Ki67 expression to see if there was a detectable increase in the number of proliferative cells in the limbus under wounding condition (figure 3.6A). Three corneas were sampled at each time point, and a total of 65 different limbal sections were stained. Ki67-nuclear positive epithelial cells in the limbus were counted and recorded as proportion of total epithelial cells in the limbal section (as determined by DAPI staining) (figure 3.6B). A one-way ANOVA found the changes in the proportion of proliferating cells to be statistically significant at the $5 \%$ level. A clear increase in the proportion of Ki67-positive cells can be observed at 24 hours and 48 hours, and this proportion returned to baseline levels by 1 week. 


\section{DISCUSSION}

A key objective of this study was to provide evidence that stem cells are located in the limbus of the porcine cornea. In doing so, this supports the use of an ex vivo model to study the behaviour of LESCs in this system. While it has been suggested that corneal epithelial stem cells may exist throughout the cornea and ocular surface (Majo et al., 2008), this conclusion and the results on which it is based has been widely challenged and found to be incompatible with the wider literature (Sun et al., 2010). Multiple lineage tracing models in mice have been developed in order to track the contribution of LESCs to epithelial maintenance. Two independent groups used confetti mouse models, in which K14+ epithelial cells, shown to be enriched in the limbus, were tracked in mice over a period of months (Amitai-Lange et al., 2015; Di Girolamo et al., 2015). In both instances it was abundantly clear that from the resulting streaks extending from the limbus to the centre, that the epithelial surface is derived primarily from progenitors in the limbus.

Functional analysis of the epithelial cells in the cornea and limbus began with single cell clonal analysis of cells from each region. The only previous published clonal analysis of the procine corneal epithelium of which the authors are aware was performed by (Majo et al., 2008), however, the authors only reported that holoclones had been identified in corneal and conjunctival populations, with no comparison to the clonal capacity of the limbal epithelium. In this study it was observed that holoclones are clearly enriched in the porcine limbal epithelium as compared with the cornea (proportion of clones classified as holoclones, $38.3 \%$ (limbus) vs. $8.3 \%$ (cornea)). The concentration of holoclones in the limbus is consistent with studies perfomed using human tissue (Pellegrini et al., 1999). In particular there is evidence that the proportion of holoclones is higher specifically within regions of the limbus with limbal crypts (Dziasko et al., 2014). However, since there is evidence that limbal crypts are present in all regions of the porcine limbus (Grieve et al., 2015), and our own results reflect this (figure 3.1A), the cells used for clonal analysis were taken from all regions of the limbus.

Notably, in human studies, holoclones have not been identified in the central cornea. However, both in this study and others (Majo et al., 2008), holoclones are shown to exist in the central cornea of the pig. While this may relate to species differences, an important factor may be the relative freshness of porcine tissue that can be obtained (within hours of death) as compared with human tissue that often has to be stored for days, or longer, before cells can be isolated. Furthermore, the tissue used in these experiments was from young pigs, whereas even fresh human tissue used for research may come from relatively much older donors, in whom the stem/progenitor cell population may be reduced. As such, freshly isolated porcine epithelial cells may retain a greater overall growth capacity in vitro. In tissue from mice it has also been shown that central corneal explants were able to generate confluent epithelial cell cultures ( $\mathrm{Li}$ et al., 2017). Therefore, it would seem likely that a population of cells may exist within the central cornea with a significant growth potential, perhaps representing transient amplifying cells or their immediate progeny.

In addition to proliferative capacity, a key stem cell property is an ability to divide slowly under resting conditions. Label retention studies have been performed on organ cultured human corneas (Figueira et al., 2007), and in vivo with mice (Li et al., 2017). In human tissue using BrdU, label-retaining epithelial cells were found in the limbus and were shown to also express putative LESC markers. In mice, however, no statistically significant difference in the frequency of label retaining cells in the limbus and cornea was observed. The results presented here align more with the results seen in humans, with roughly 10 times as many label-retaining cells (44.7 76.4 vs. $4.7 \pm 1.5)$ cells being identified in limbal sections. This result is supported by gene expression studies of the porcine limbal side population, in which the molecular signature identified a phenotype characterised by slow cycling and low metabolic activity (Akinci et al., 2009).

The presence of holoclone generating cells within the central cornea may reflect the findings in other selfrenewing tissues, which suggest that multiple populations of stem cells may exist (Arwert et al., 2012; Tian et al., 2011). However, the absence of a unifying marker or assay identifying LESCs is a significant and currently unresolved challenge within the field and as such, the presence of holoclones alone cannot confirm the presence of epithelial stem cells in the central cornea. Therefore, the results of all of the studies presented here must be taken together. The exclusive expression of putative LESC markers (p63 and CK15) in the porcine limbal epithelium, (in agreement with results presented by (Notara et al., 2011)) in addition to the results of the clonal 
analysis and label retention studies strongly supports the view that LESCs exist, and are enriched in the porcine limbal epithelium. This strongly mirrors the studies of human tissue, and the authors conclude that porcine tissue therefore represents a suitable and useful model for studying the behaviour of human LESCs.

Researchers have been taking advantage of the ease with which corneal tissue can be maintained under organ culture conditions for decades. The corneal organ culture models that have been previously developed have been very valuable in many studies and have been of enormous help for other researchers wishing to develop their own models. Importantly, it is clear that different groups have approached this problem in a range of ways, and this ultimately reflects the investigative objectives. For example, in groups wishing to perform studies on, or involving, the endothelial surface of the cornea, more complex culture systems have been developed in which the epithelium and endothelium are independently and constantly fed and irrigated (Zhao et al).

For studies in which the focus is primarily on the behaviour of the epithelial surface simpler models have been used. The earliest models dealt with the issue of maintaining the anterior chamber structure by filling the concavity with an agar mixture (Foreman et al., 1996). While much of the design has remained unchanged, other interventions have been introduced including rocking of the tissue in culture to intermittently expose the tissue to air and liquid environments. This has been shown to lead to improvements in corneal morphology, and the duration for which corneas can be kept in culture (Deshpande et al., 2015; Richard et al., 1991).

In the organ culture system used here, the corneoscleral rims were filled with agar, and also kept on an orbital 3D-rotating shaker within the incubator. However, an additional feature, 3D-printed platforms, were introduced to help keep the cornea/agar complex in a single position. It was observed that if the cornea/agar complex slid around too much in the well, there was an increased likelihood that the cornea would detach from the agar and compromise the model. By attaching the agar to the pegs on the platform, the tissue was kept in a single position in the well over long periods, despite the constant movement on the rotating platform.

With respect to the media used in this study, the use of serum should certainly be considered when interpreting the results. Fetal bovine serum is a nutritionally dense additive which may have wide ranging effects on transcription and cell behaviour. It has been demonstrated that while serum-containing media didn't significantly alter wound healing rates, its addition resulted in a much improved cellular morphology (Proietto et al., 2017). Since one of the outcomes of this study was assessing the profile of putative LESC marker expression in tissue following organ culture, it was considered to be important to include serum in the media to support the normal cellular microstructure.

Early observations with the organ culture system presented here demonstrated that if corneas were wounded at the same time as being put into culture, there was a great deal of variation in re-epithelialisation, with a small proportion of corneas failing to regenerate the epithelial surface. In response, a pre-treatment phase was implemented to exclude corneas that did not demonstrate regeneration of the epithelial surface to confirm a conserved baseline function of the tissues. The advantage of this preliminary step can be clearly observed in the consistent and replicable rates of epithelial wound healing that were observed.

The authors appreciate that a more representative picture of the in vivo tissue physiology would be preserved if the time spend in culture is minimised. Changes following enucleation and organ culture of the corneoscleral rim include the loss of a functional neurovascular supply. Studies with mouse corneas has shown that sub-basal and deeper stromal nerves begins to disassemble a matter of hours after enucleation and organ culture (Stepp et al., 2014). The integrity of nerve fibres to be important for sensing certain stimuli, leading to the release of essential neurotrophins for corneal homeostasis and wound healing (Pal-Ghosh et al., 2017). Additionally, the loss of blood flow through the limbal vessels prevents the contribution of humoral components like immune cells and other factors that may play roles in mediating corneal wound healing, as well as a preservation of a functional stem cell population (Ljubimov and Saghizadeh, 2015).

Despite these limitations, with the organ culture model presented here, we have observed that both the limbal crypt microarchitecture and the pattern of staining for CK15 and epithelial differentiation marker CK3 are maintained. The only difference seen in the organ culture corneas was the fact that in addition to expression in the basal limbus, p63 was found to be expressed in basal cells of the central corneal epithelium. This observation is consistent with previous observations in 'activated' human corneal tissue (Di Iorio et al., 2005). It may also reflect changes in the stiffness of the central corneal tissue, which becomes softer under organ culture 
conditions due to swelling (Gouveia et al., 2019). The label retention studies which were carried out over four weeks in organ cultured corneas demonstrate an intact ability of limbal epithelial cells to divide slowly, a hallmark of progenitor cell function. These also support the idea that there is preservation of the phenotype of the basal limbal epithelium in which LESCs are believed to reside.

Furthermore, in this model we observed that there was an increase in the proportion of Ki67-positive cells in the limbus following central corneal wounding. The increase was most evident at 24- and 48-hours following wounding, and this appeared to resolve to baseline by 1-week post-wounding. Together with evidence of the requirement for an intact niche for epithelial regeneration, as demonstrated by the failure of all N-heptanol treated tissues to re-epithelialise at all, this strongly supports the functionality and necessity for an intact limbal niche in this model.

The time delay in this response would appear to be consistent with observations in human organ culture corneal wounding studies, where in the first 12 hours following wound creation, no proliferative response in detected (Chang et al., 2008). Since there is closure of the wound in this model preceding the proliferative response seen in the limbus, it is thought that a limbal-independent phase of wound healing, where central corneal epithelial cells at the wound edge spread and migrate to cover the surface, is present. The choice of a large $8 \mathrm{~mm}$ epithelial wound was made in order to stimulate a limbal response, as studies have suggested that perhaps there is no limbal involvement in the close of relatively smaller central wounds (Amitai-Lange et al., 2015; Majo et al., 2008).

\section{CONCLUSION}

The results shown here build on our current understanding of the porcine corneal epithelial phenotype and its suitability as a model of the human corneal epithelium. Furthermore, we demonstrate that a simple organ culture method, that preserves limbal epithelial phenotype can be used for studies of the role of the limbus in corneal epithelial wound healing. Critically, the presence of key niche factors in this 3D tissue make it a practical and powerful tool for gaining further insight into the factors that regulate LESC fate and allow us to further contextualise results from studying cellular behaviour in $2 \mathrm{D}$ in vitro systems. 


\section{FIGURES}

A
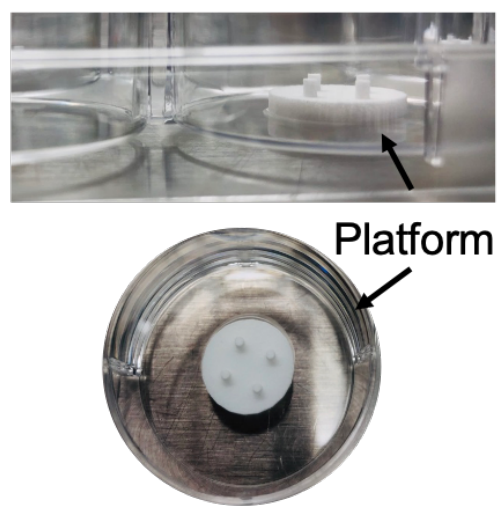

C

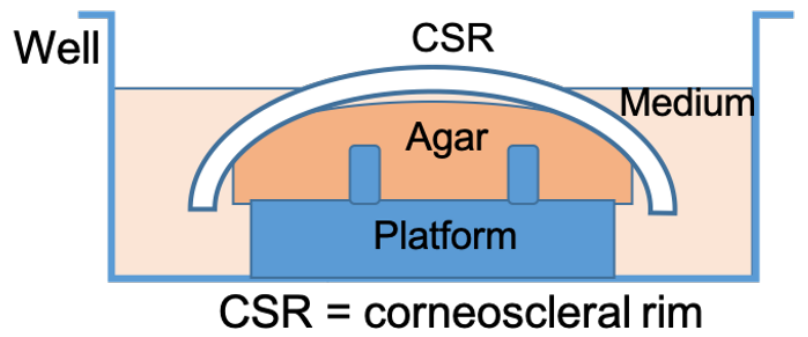

B

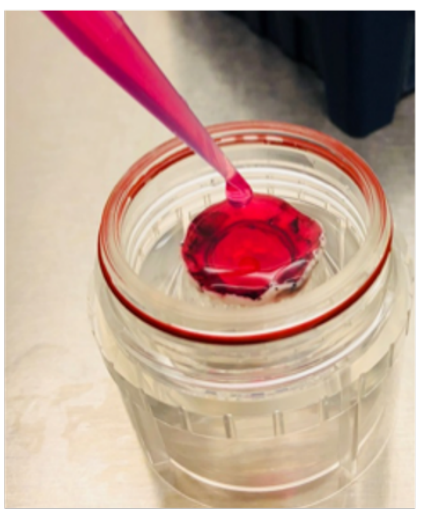

D

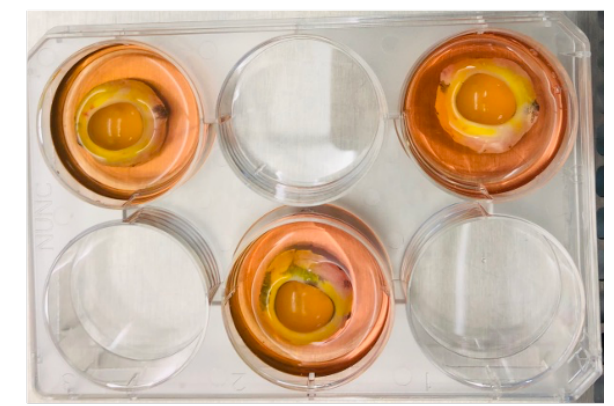

Figure 2.1

(A) A small platform was designed and 3D-printed in autoclavable material (nylon), and was attached to the bottom of sterile 6-well plates with cyanoacrylate glue (B) Once washed, corneoscleral rims were inverted and filled with warmed liquid agar mixture which was left to set (C) A schematic of the organ culture system in which the agar filled corneoscleral rim was tethered to the well via the pegs sticking up from the 3D printed platform. This stabilised the tissue when kept on the orbital shaker within the incubator, preventing it from separating from the agar. Medium was filled to the level of the limbus, and the rotation motion allowed it to wash over the surface of the cornea to substitute the action of the tear film (D) Three eyes in the organ culture system on a single modified 6-well plate. 
A

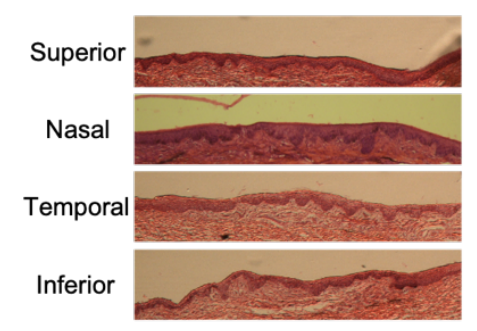

C
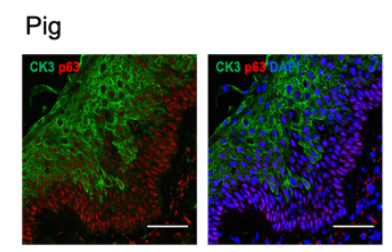

Human

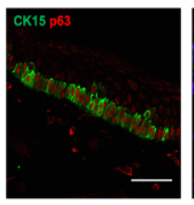

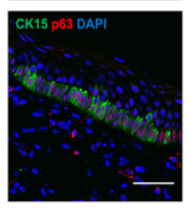
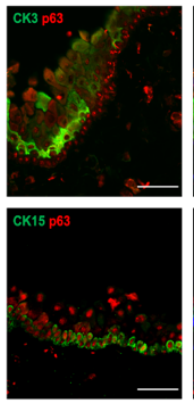

B
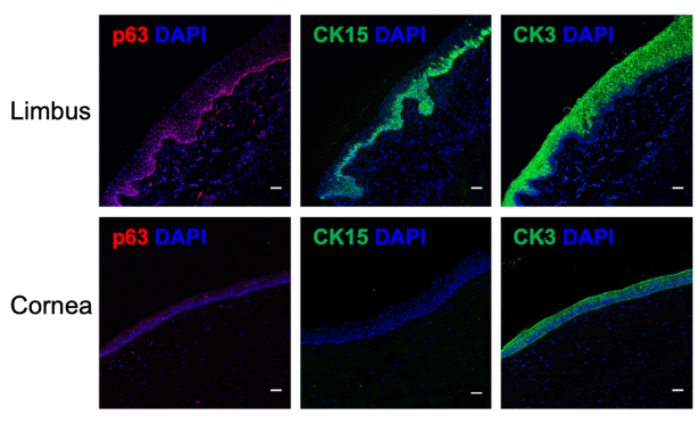

D
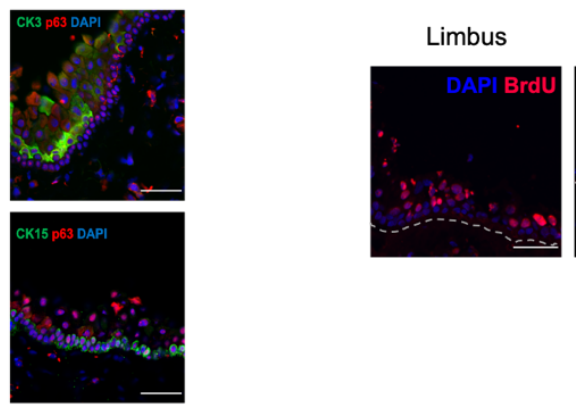

Cornea

Cornea
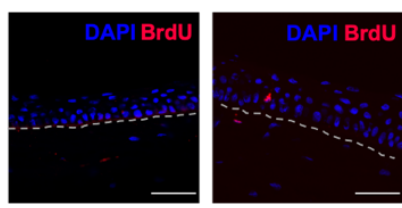

Figure 3.1

(A) Limbal interpalisade crypt-like structures were identified in all quadrants of the porcine cornea (B) Immunofluorescence staining revealed that p63 and cytokeratin 15 were expressed in the basal layers of the limbus, and not the central cornea, while cytokeratin 3 was expressed in the suprabasal and superficial layers of the limbus, and throughout the entire corneal epithelium (C) Comparison of the staining patters of the human and porcine limbus, with the expression patterns of p63, cytokeratin 15, and cytokeratin 3 being conserved (D) Label-retaining cells staining for BdrU after a 4 week chase period are primarily found in the limbus. Dotted line indicates junction between the epithelium and stroma. Scalebar $=50 \mu m . n=3$.

A

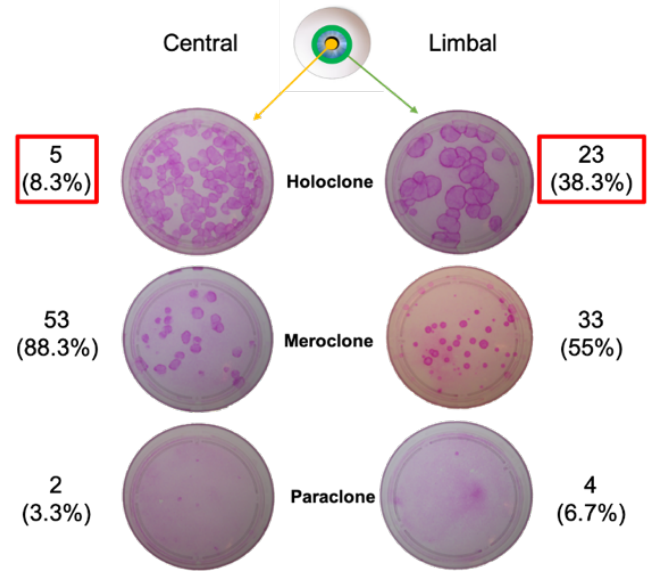

B

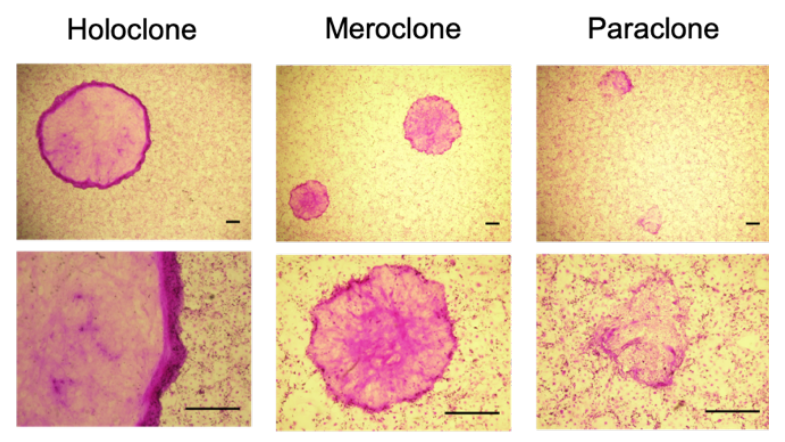

Figure 3.2

(A) The proportion of holoclones, meroclones, and paraclones identified in the 60 clones taken from either the central or corneal epithelium of three different pigs. A significantly higher number of holoclones were identified in the limbal region as compared with the central corneal epithelium (B) Images detailing the typical appearance of colonies found on plates identified as holoclones, meroclones, or paraclones based on the proportion of terminal colonies. Holoclones show a typically large size, smooth rounded edge and small cells throughout. Scalebar $=500 \mu \mathrm{m}$ 
A

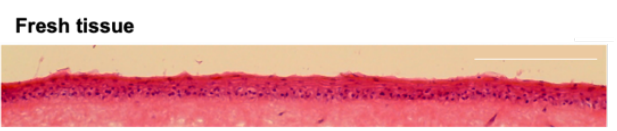

Organ culture conditions

B
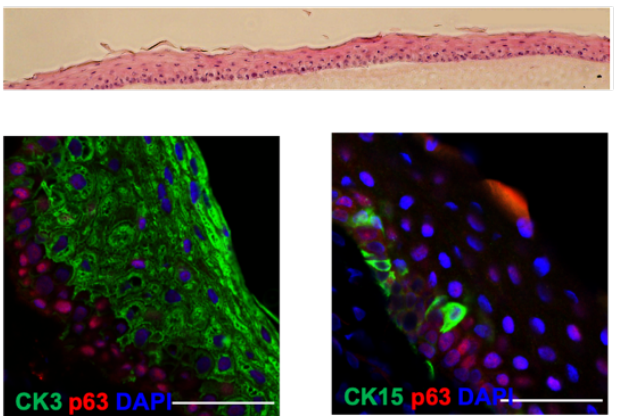

C

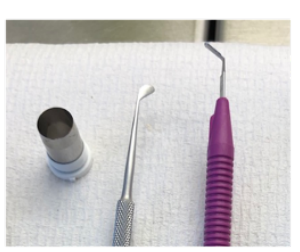

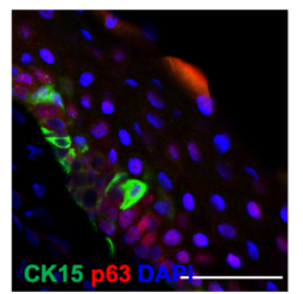

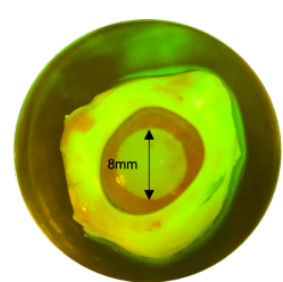

D

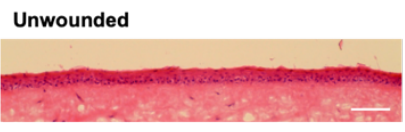

Debrided wound edge

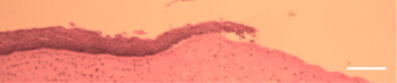

Regenerating wound edge

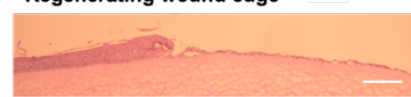

Regenerating central cornea

$E$
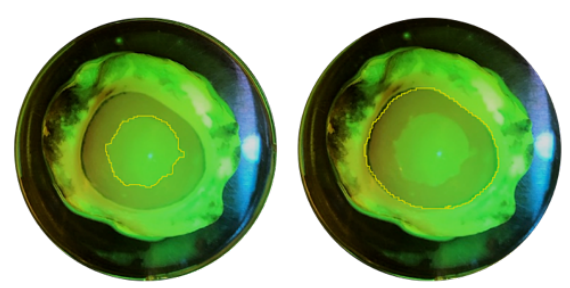

Figure 3.3

(A) Under organ culture conditions, H\&E staining shows the preservation of a multi-layered epithelium

(B) Immunofluorescence staining demonstrating that $\mathrm{p63}$ and cytokeratin 15 staining of the basal layer of the limbus, and cytokeratin 3 staining of the suprabasal and superficial layers is preserved under organ culture conditions (C) Epithelial wounds were created using an $8 \mathrm{~mm}$ diameter trephine to demarcate the wound area, and the debridement was performed using a paediatric crescent and hockey stick tool. An $8 \mathrm{~mm}$ wound area was selected to ensure a limbal response to the epithelial injury (D) H\&E staining was performed to confirm the selective removal of the epithelium following debridement, and an intact basement membrane. Sections of healing tissues were also stained demonstrating a growing monolayer of epithelium in the wound healing response, which was later followed by stratification (E) The size of the wound was monitored by measuring the size of the stained area as a proportion of the total corneal area in each image using ImageJ software. Scalebar $=50 \mu m$ 
A

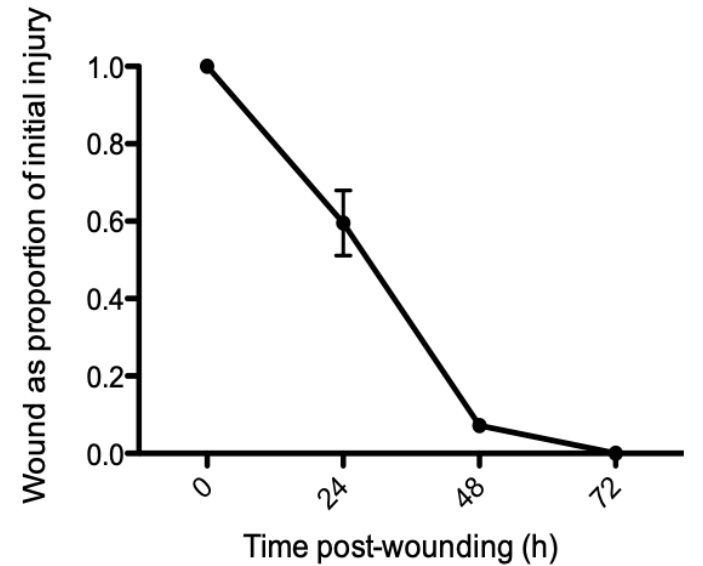

B

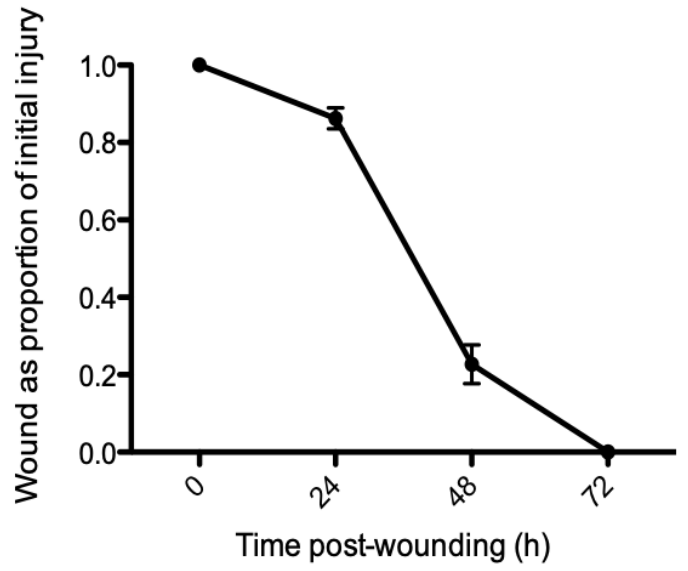

C

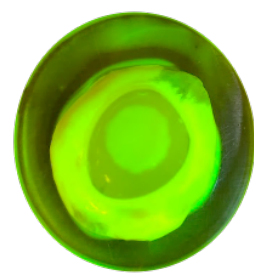

Oh

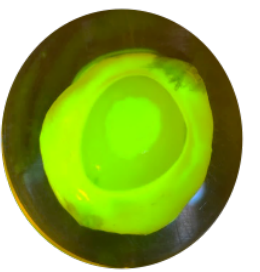

$24 h$

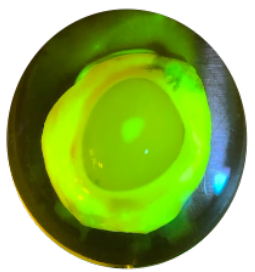

48h

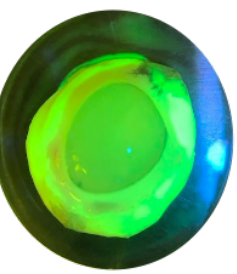

72h

Figure 3.4

(A) The rate of closure of an $8 \mathrm{~mm}$ epithelial wound in 5 different organ cultured eyes. The greatest variation was seen in the first 24 hours, but the time courses converge by 48 hours, and all eyes have completely resurfaced by 72 hours (B) A single eye was wounded $(8 \mathrm{~mm})$ on three consecutive occasions and the time course of wound healing was observed to be consistent each time (C) An example of the typical size of the staining area in an eye following the creation of a central epithelial wound over 72 hours. Where error bars are not visible on graphs, SEM was too small to register on this size of image. 
A

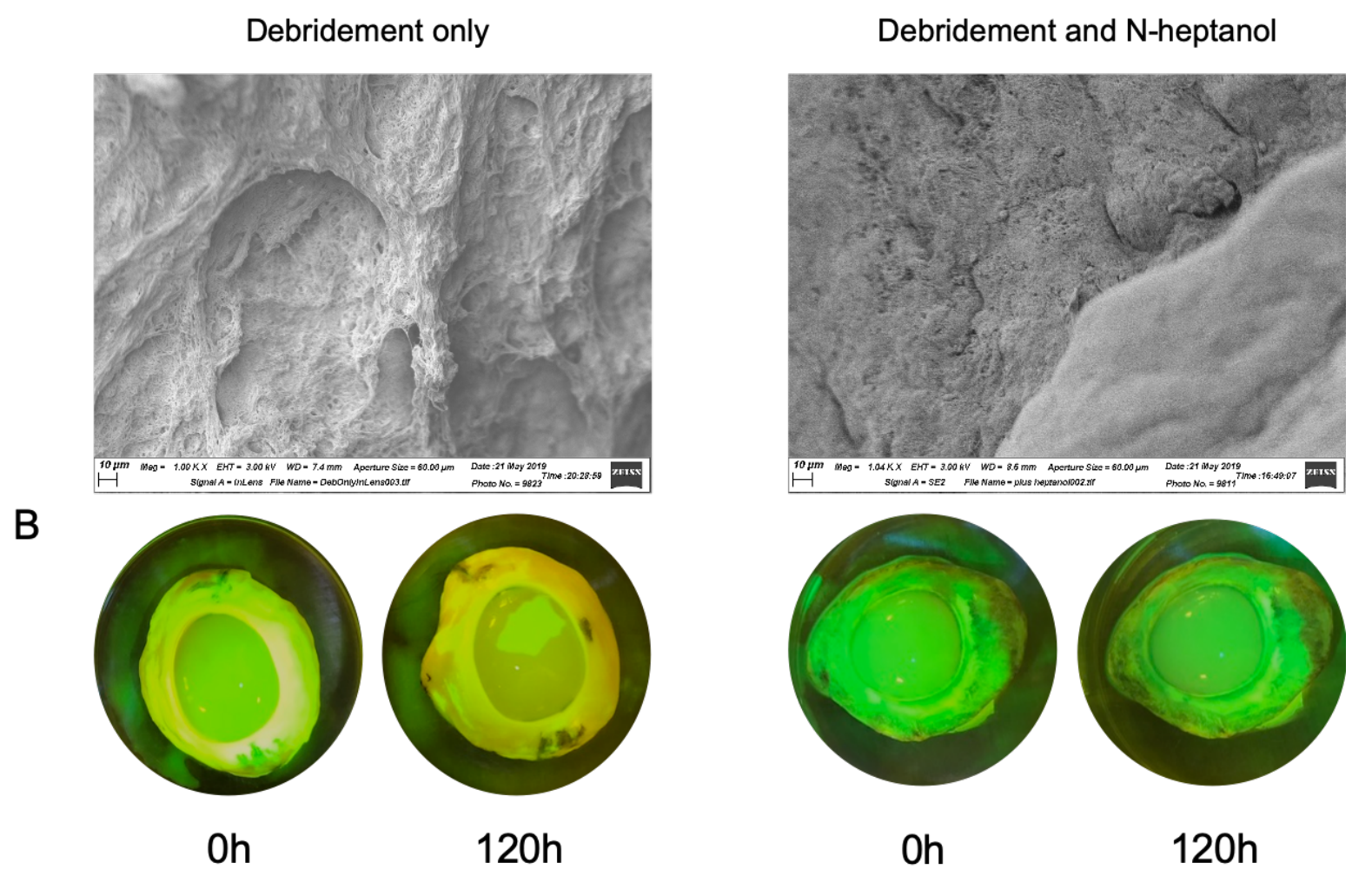

Figure 3.5

(A) SEM images (1000X magnification) of the limbus following either debridement alone, or debridement followed by 120 s of treatment with $1 \mathrm{M} \mathrm{N}$-heptanol. In the debridement only tissue, the interpalisade crypt structures are clearly intact. Following treatment with $\mathrm{N}$-heptanol, there is a loss of the crypt-like microarchitecture, and a "burned" appearance (B) In the eyes on the left, the entire surface of the cornea and the limbus was debrided, and within 5 days, epithelium regenerated from the limbus towards the centre. In the eyes on the right, the entire corneal and limbal surface was debrided, followed by selective treatment of the limbus with $\mathrm{N}$-heptanol soaked paper (cut out into a ring) for 120s. In the latter tissue, there was no evidence of epithelial regeneration across the entire ocular surface. $(n=4)$. 
A

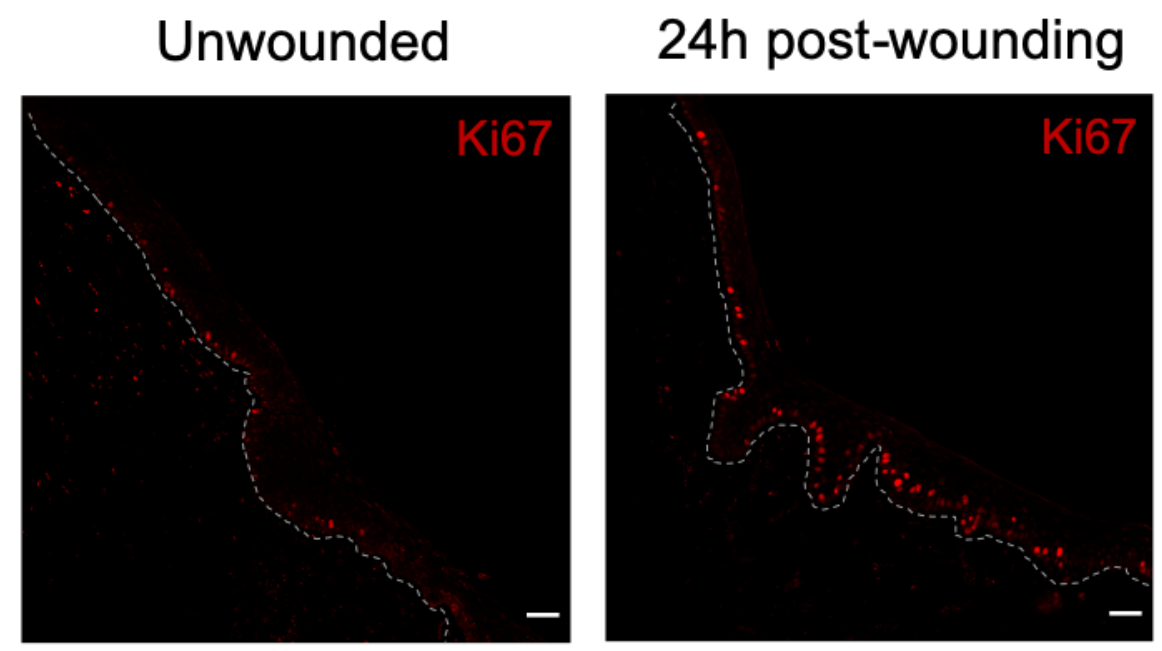

B

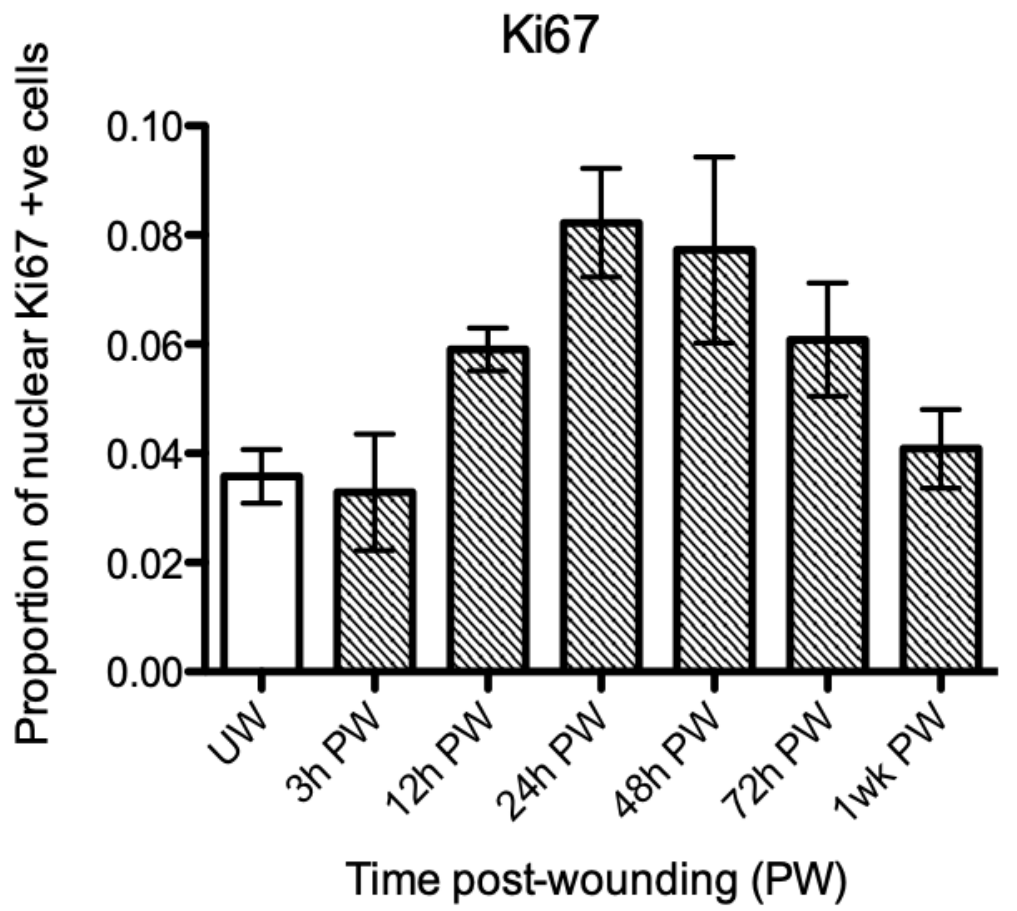

Figure 3.6

Following the creation of an $8 \mathrm{~mm}$ central epithelial wound, eyes were frozen and sectioned at different time points (A) Side by side of the Ki67 staining pattern in the limbal section of unwounded tissue, and limbal tissue frozen at $24 \mathrm{~h}$ post-wounding. Dotted line indicates junction between the epithelium and stroma (B) Sections taken from tissue ( $n=3$ at each time point) at a range of time points, up to one week were stained for Ki67, and the proportion of all epithelial cells as stained with DAPI that were nuclear positive for Ki67 were counted. Scalebar $=50 \mu \mathrm{m}$ 


\section{REFERENCES}

Akinci, M.A.M., Turner, H., Taveras, M., Wolosin, J.M., 2009. Differential gene expression in the pig limbal side population: implications for stem cell cycling, replication, and survival. Invest. Ophthalmol. Vis. Sci. 50, 5630-5638. https://doi.org/10.1167/iovs.09-3791

Amitai-Lange, A., Altshuler, A., Bubley, J., Dbayat, N., Tiosano, B., Shalom-Feuerstein, R., 2015. Lineage tracing of stem and progenitor cells of the murine corneal epithelium. Stem Cells 33, 230-239. https://doi.org/10.1002/stem. 1840

Arwert, E.N., Hoste, E., Watt, F.M., 2012. Epithelial stem cells, wound healing and cancer. Nature Reviews Cancer 12, 170-180. https://doi.org/10.1038/nrc3217

Barrandon, Y., Green, H., 1987. Three clonal types of keratinocyte with different capacities for multiplication. Proc. Natl. Acad. Sci. U.S.A. 84, 2302-2306.

Barrera, V., Troughton, L.D., Iorio, V., Liu, S., Oyewole, O., Sheridan, C.M., Hamill, K.J., 2018. Differential Distribution of Laminin N-Terminus a 31 Across the Ocular Surface: Implications for Corneal Wound Repair. Invest. Ophthalmol. Vis. Sci. 59, 4082-4093. https://doi.org/10.1167/iovs.18-24037

Chang, C.-Y., Green, C.R., McGhee, C.N.J., Sherwin, T., 2008. Acute wound healing in the human central corneal epithelium appears to be independent of limbal stem cell influence. Invest. Ophthalmol. Vis. Sci. 49, 5279-5286. https://doi.org/10.1167/iovs.07-1260

Crespo-Moral, M., García-Posadas, L., López-García, A., Diebold, Y., 2020. Histological and immunohistochemical characterization of the porcine ocular surface. PLoS One 15. https://doi.org/10.1371/journal.pone.0227732

Deshpande, P., Ortega, Í., Sefat, F., Sangwan, V.S., Green, N., Claeyssens, F., MacNeil, S., 2015. Rocking Media Over Ex Vivo Corneas Improves This Model and Allows the Study of the Effect of Proinflammatory Cytokines on Wound Healing. Invest. Ophthalmol. Vis. Sci. 56, 1553-1561. https://doi.org/10.1167/iovs.14-15308

Di Girolamo, N., Bobba, S., Raviraj, V., Delic, N.C., Slapetova, I., Nicovich, P.R., Halliday, G.M., Wakefield, D., Whan, R., Lyons, J.G., 2015. Tracing the fate of limbal epithelial progenitor cells in the murine cornea. Stem Cells 33, 157-169. https://doi.org/10.1002/stem.1769

Di Iorio, E., Barbaro, V., Ruzza, A., Ponzin, D., Pellegrini, G., De Luca, M., 2005. Isoforms of DeltaNp63 and the migration of ocular limbal cells in human corneal regeneration. Proc. Natl. Acad. Sci. U.S.A. 102, 9523-9528. https://doi.org/10.1073/pnas.0503437102

Dorà, N.J., Hill, R.E., Collinson, J.M., West, J.D., 2015. Lineage tracing in the adult mouse corneal epithelium supports the limbal epithelial stem cell hypothesis with intermittent periods of stem cell quiescence. Stem Cell Res 15, 665-677. https://doi.org/10.1016/j.scr.2015.10.016

Dziasko, M.A., Armer, H.E., Levis, H.J., Shortt, A.J., Tuft, S., Daniels, J.T., 2014. Localisation of Epithelial Cells Capable of Holoclone Formation In Vitro and Direct Interaction with Stromal Cells in the Native Human Limbal Crypt. PLOS ONE 9, e94283. https://doi.org/10.1371/journal.pone.0094283

Dziasko, M.A., Daniels, J.T., 2016. Anatomical Features and Cell-Cell Interactions in the Human Limbal Epithelial Stem Cell Niche. Ocul Surf 14, 322-330. https://doi.org/10.1016/j.jtos.2016.04.002

Figueira, E.C., Di Girolamo, N., Coroneo, M.T., Wakefield, D., 2007. The phenotype of limbal epithelial stem cells. Invest. Ophthalmol. Vis. Sci. 48, 144-156. https://doi.org/10.1167/iovs.06-0346

Foreman, D.M., Pancholi, S., Jarvis-evans, J., McLEOD, D., Boulton, M.E., 1996. A Simple Organ Culture Model for Assessing the Effects of Growth Factors on Corneal Re-epithelialization. Experimental Eye Research 62, 555-564. https://doi.org/10.1006/exer.1996.0065

Gouveia, R.M., Lepert, G., Gupta, S., Mohan, R.R., Paterson, C., Connon, C.J., 2019. Assessment of corneal substrate biomechanics and its effect on epithelial stem cell maintenance and differentiation. Nature Communications 10, 1-17. https://doi.org/10.1038/s41467-019-09331-6

Grieve, K., Ghoubay, D., Georgeon, C., Thouvenin, O., Bouheraoua, N., Paques, M., Borderie, V.M., 2015. Three-dimensional structure of the mammalian limbal stem cell niche. Exp. Eye Res. 140, 75-84. https://doi.org/10.1016/j.exer.2015.08.003

Li, J., Xiao, Y., Coursey, T.G., Chen, X., Deng, R., Lu, F., Pflugfelder, S.C., Li, D.-Q., 2017. Identification for Differential Localization of Putative Corneal Epithelial Stem Cells in Mouse and Human. Sci Rep 7. https://doi.org/10.1038/s41598-017-04569-w

Ljubimov, A.V., Saghizadeh, M., 2015. Progress in corneal wound healing. Prog Retin Eye Res 49, 17-45. https://doi.org/10.1016/j.preteyeres.2015.07.002

Lydersen, S., Pradhan, V., Senchaudhuri, P., Laake, P., 2007. Choice of test for association in small sample unorderedr $\times c$ tables. Statist. Med. 26, 4328-4343. https://doi.org/10.1002/sim.2839 
Majo, F., Rochat, A., Nicolas, M., Jaoudé, G.A., Barrandon, Y., 2008. Oligopotent stem cells are distributed throughout the mammalian ocular surface. Nature 456, 250-254. https://doi.org/10.1038/nature07406

Notara, M., Schrader, S., Daniels, J.T., 2011. The porcine limbal epithelial stem cell niche as a new model for the study of transplanted tissue-engineered human limbal epithelial cells. Tissue Eng Part A 17, 741750. https://doi.org/10.1089/ten.TEA.2010.0343

Pal-Ghosh, S., Tadvalkar, G., Stepp, M.A., 2017. Alterations in Corneal Sensory Nerves During Homeostasis, Aging, and After Injury in Mice Lacking the Heparan Sulfate Proteoglycan Syndecan-1. Invest Ophthalmol Vis Sci 58, 4959-4975. https://doi.org/10.1167/iovs.17-21531

Patruno, M., Perazzi, A., Martinello, T., Blaseotto, A., Di Iorio, E., Iacopetti, I., 2017. Morphological description of limbal epithelium: searching for stem cells crypts in the dog, cat, pig, cow, sheep and horse. Vet. Res. Commun. 41, 169-173. https://doi.org/10.1007/s11259-017-9676-y

Pellegrini, G., Golisano, O., Paterna, P., Lambiase, A., Bonini, S., Rama, P., De Luca, M., 1999. Location and Clonal Analysis of Stem Cells and Their Differentiated Progeny in the Human Ocular Surface. J Cell Biol 145, 769-782.

Proietto, L.R., Whitley, R.D., Brooks, D.E., Schultz, G.E., Gibson, D.J., Berkowski, W.M., Salute, M.E., Plummer, C.E., 2017. Development and Assessment of a Novel Canine Ex Vivo Corneal Model. Curr. Eye Res. 42, 813-821. https://doi.org/10.1080/02713683.2016.1262428

Richard, N.R., Anderson, J.A., Weiss, J.L., Binder, P.S., 1991. Air/liquid corneal organ culture: a light microscopic study. Current Eye Research 10, 739-749. https://doi.org/10.3109/02713689109013868

Saghizadeh, M., Kramerov, A.A., Yu, F.X., Castro, M.G., Ljubimov, A.V., 2010. Normalization of Wound Healing and Diabetic Markers in Organ Cultured Human Diabetic Corneas by Adenoviral Delivery of c-Met Gene. Invest Ophthalmol Vis Sci 51, 1970-1980. https://doi.org/10.1167/iovs.09-4569

Shortt, A.J., Secker, G.A., Munro, P.M., Khaw, P.T., Tuft, S.J., Daniels, J.T., 2007. Characterization of the limbal epithelial stem cell niche: novel imaging techniques permit in vivo observation and targeted biopsy of limbal epithelial stem cells. Stem Cells 25, 1402-1409. https://doi.org/10.1634/stemcells.2006-0580

Stepp, M.A., Zieske, J.D., Trinkaus-Randall, V., Kyne, B., Pal-Ghosh, S., Tadvalkar, G., Pajoohesh-Ganji, A., 2014. Wounding the Cornea to Learn How it Heals. Exp Eye Res 121, 178-193. https://doi.org/10.1016/j.exer.2014.02.007

Sun, T.-T., Tseng, S.C., Lavker, R.M., 2010. Location of corneal epithelial stem cells. Nature 463, E10.

Tannenbaum, J., Bennett, B.T., 2015. Russell and Burch's 3Rs Then and Now: The Need for Clarity in Definition and Purpose. J Am Assoc Lab Anim Sci 54, 120-132.

Thoft, R.A., Friend, J., 1983. The X, Y, Z hypothesis of corneal epithelial maintenance. Invest. Ophthalmol. Vis. Sci. 24, 1442-1443.

Tian, H., Biehs, B., Warming, S., Leong, K.G., Rangell, L., Klein, O.D., de Sauvage, F.J., 2011. A reserve stem cell population in small intestine renders Lgr5-positive cells dispensable. Nature 478, 255-259. https://doi.org/10.1038/nature10408

Yazdanpanah, G., Haq, Z., Kang, K., Jabbehdari, S., Rosenblatt, M.L., Djalilian, A.R., 2019. Strategies for reconstructing the limbal stem cell niche. Ocul Surf 17, 230-240. https://doi.org/10.1016/j.jtos.2019.01.002

Zhao, B., Cooper, L.J., Brahma, A., MacNeil, S., Rimmer, S., Fullwood, N.J., 2006. Development of a ThreeDimensional Organ Culture Model for Corneal Wound Healing and Corneal Transplantation. Invest. Ophthalmol. Vis. Sci. 47, 2840-2846. https://doi.org/10.1167/iovs.05-1367

\section{REFERENCES}

The authors would like to gratefully acknowledge the funding provided by the Rosetrees foundation that supported this work. 